\title{
Microstructural Characterization of Resistance Artery Remodelling in Diabetes Mellitus
}

\author{
James S. Bella,b,c Aminat O. Adio ${ }^{d}$ Andrew Pitt $^{d}$ Lindsay Hayman ${ }^{d}$ \\ Clare E. Thorn $^{d}$ Angela C. Shore ${ }^{d}$ Jacqueline L. Whatmore ${ }^{b}$ C. Peter Winlove ${ }^{a}$ \\ ${ }^{a}$ Department of Physics, University of Exeter, Exeter, UK; b Institute of Biomedical and Clinical Science, University of \\ Exeter Medical School, University of Exeter, Exeter, UK; 'SChool of Optometry and Vision Sciences, Cardiff University, \\ Cardiff, UK; ${ }^{d}$ Diabetes and Vascular Medicine, Institute of Biomedical and Clinical Sciences, University of Exeter \\ Medical School and NIHR Exeter Clinical Research Facility, Exeter, UK
}

\author{
Keywords \\ Diabetes mellitus · Collagen · Elastin · Remodelling · \\ Multiphoton microscopy
}

\begin{abstract}
Introduction: Microvascular remodelling is a symptom of cardiovascular disease. Despite the mechanical environment being recognized as a major contributor to the remodelling process, it is currently only understood in a rudimentary way. Objective: A morphological and mechanical evaluation of the resistance vasculature in health and diabetes mellitus. Methods: The cells and extracellular matrix of human subcutaneous resistance arteries from abdominal fat biopsies were imaged using two-photon fluorescence and second harmonic generation at varying transmural pressure. The results informed a two-layer mechanical model. Results: Diabetic resistance arteries reduced in wall area as pressure was increased. This was attributed to the presence of thick, straight collagen fibre bundles that braced the outer wall. The abnormal mechanical environment caused the internal elastic lamina and endothelial and vascular smooth muscle cell arrangements to twist. Conclusions: Our results suggest diabetic microvascular remodelling is
\end{abstract}

likely to be stress-driven, comprising at least 2 stages: (1) Laying down of adventitial bracing fibres that limit outward distension, and (2) Deposition of additional collagen in the media, likely due to the significantly altered mechanical environment. This work represents a step towards elucidating the local stress environment of cells, which is crucial to build accurate models of mechanotransduction in disease.

(c) 2021 The Author(s)

Published by S. Karger AG, Basel

\section{Introduction}

Diabetes mellitus is a disease that affects 1 in 11 people worldwide, and its prevalence is increasing [1]. Microvascular remodelling is a complication of diabetes [2], as well as hypertension [3] and obesity [4], which results in altered arterial wall structure and a loss of vasoactive responsivity. The network of small arteries is the primary regulator of tissue perfusion, and compromising their vasoactive ability can contribute to clinical problems such as retinopathy and cardiovascular disease.

Changes in the structure of small arteries and their gross mechanical properties in disease have been described in some detail (reviewed in e.g., [5]). In general,
(C) 2021 The Author(s)

Published by S. Karger AG, Basel

This is an Open Access article licensed under the Creative Commons Attribution-NonCommercial-4.0 International License (CC BY-NC) (http://www.karger.com/Services/OpenAccessLicense), applicable to the online version of the article only. Usage and distribution for commercial purposes requires written permission.
Correspondence to:

James S. Bell, bellj10@ cardiff.ac.uk 
inward remodelling is associated with essential hypertension, whereby the inner and outer diameters are reduced, and the ratio of wall thickness to lumen diameter increases, but the wall volume is conserved [6]. More advanced remodelling can involve cell hypertrophy and fibrosis, whereby additional collagen is deposited in the vessel wall [7]. In diabetes, small artery pathology is worsened by the presence of other cardiovascular pathologies, such as hypertension. Normotensive individuals with diabetes or obesity have been reported to exhibit significant hypertrophy [8], but while obese individuals exhibit no change in homogeneous wall stiffness, chronic hyperglycaemia in diabetes can lead to the creation of advanced glycosylation end products, which have the effect of cross-linking and stiffening extracellular matrix proteins in the vascular wall [6].

What is currently lacking is an understanding of the microstructural bases of these changes and their association with the micromechanics of the tissue. This level of understanding is important to distinguish causal from compensatory changes, to test hypotheses on the role of changes in cellular mechanotransduction (see e.g., [9]) and to target therapeutic approaches. However, this is a challenging task because of the complexity of the relationships between structure and micromechanics, which are only now becoming apparent in large vessels $[10,11]$.

Human small resistance arteries (diameter $100 \mu \mathrm{m}$ to approx. $400 \mu \mathrm{m})$ are, like their larger counterparts, arranged in 3 layers. The intima comprises endothelial cells and elastic fibres, the media is highly cellularized and comprises vascular smooth muscle cells (VSMCs) interspersed by a small quantity of thin elastic fibres, and the adventitia comprises fibroblasts, bundles of fibrous collagen, and a network of elastic fibres. In normal vessels, the overall pressure-deformation characteristics have been described in terms of serial "hook-on" models [12], based upon the principle that elastic structures allow large-scale distension arrested by collagen fibres that recruit $[13,14]$ to prevent damaging overextension $[15,16]$. On a microscopic scale, recent micromechanical studies [17-20] have demonstrated complex interactions between the collagen and elastin networks within each layer in response to changes in luminal pressure, suggesting simple quantification using, for instance, histological techniques belies the true mechanical environment.

The aim of the present work was to investigate how the microstructure of small arteries and their passive response to transmural pressure is modified by vascular remodelling associated with normotensive type 2 diabetes mellitus (T2DM). Small subcutaneous resistance arteries from control subjects and T2DM volunteers were investigated in the passive state using a combination of myography and multiphoton microscopy, following protocols we have described recently [17]. Significant differences were found in the morphology of the elastic fibre and collagen networks, and mechanical properties between the 2 populations. These differences could be broadly recognized as contributing to inward remodelling or fibrosis.

\section{Materials and Methods}

Biopsies were obtained from 21 volunteers in the Exeter 10,000 cohort, of whom 13 were healthy (control group), and 8 had T2DM. Exclusion criteria were as follows: Blood pressure exceeding 140/90 mm Hg; treatment with insulin, GLP-1 analogues, oral steroids, or anti-inflammatory drugs, including aspirin; pregnancy; lactating women; endocrine disorders; $\mathrm{HbA} 1 \mathrm{C}>75 \mathrm{mmol} / \mathrm{mol}$; history of acute MI or cerebrovascular disease in the previous 6 months; current treatment with warfarin or history of any bleeding disorders. The T2DM group had HbA1C values of $58.3 \pm 8.7$ $\mathrm{mmol} / \mathrm{mol}$ and had been living with T2DM for $11.4 \pm 2.3$ years (range 9-15 years). Two individuals with diabetes had long standing treated hypertension. There was no history of heart disease, including angina, peripheral vascular disease, or previous angioplasty within the group. The T2DM group had the following complications: 2 cases of neuropathy, 1 case of retinopathy (treated with laser therapy), and no nephropathy. A local anaesthetic was applied prior to obtaining an abdominal adipose tissue biopsy by scalpel incision at $10 \mathrm{~cm}$ laterally to the right of the umbilicus. The excised tissue was transported immediately to the laboratory for imaging. Laboratory scientists and data analysts were blind to the state of health of the sample donors.

The experimental procedure has been described in detail previously [17] and is illustrated in Figure 1. Briefly, resistance arteries of outer diameter $100-400 \mu \mathrm{m}$ were dissected from the adipose tissue taking care to leave the adventitia intact. Segments 3-5 mm in length with no side branches were transferred carefully to a custom-made myograph. Biopsies and dissected arteries were immersed at all times in 3-[N-morpholino] propane sulfonic acid buffer adjusted to a $\mathrm{pH}$ of $7.40 \pm 0.02$, at $4^{\circ} \mathrm{C}$.

The dissected resistance arteries were cannulated on the myograph with glass capillaries of diameter approximately $20 \mu \mathrm{m}$ and secured with 11-0 gauge suture (Ethicon). The resistance arteries were perfused to remove blood, taking care to prevent introducing bubbles, and then straightened by moving the capillary tubes apart slowly. The myograph was then transferred to the stage of a multiphoton microscope, which included the temperature control for the myograph bath and transmural pressure control (kept at $3 \mathrm{~mm}$ $\mathrm{Hg}$ to maintain vessel shape). With the myograph bath maintained at $37^{\circ} \mathrm{C}$, high resolution 3 -dimensional (3D) image stacks of a portion of the vessel wall were acquired at 3, 10, 20, 30, and $50 \mathrm{~mm} \mathrm{Hg}$ transmural pressures, allowing $15 \mathrm{~min}$ of equilibration after each pressure increment before imaging. The pressure increments were chosen for 3 reasons: (i) consistency with a previous study [17]; (ii) the most significant morphological changes associated with vascular remodelling occur within the extracellular matrix, which contributes only to the passive mechanical response, and the majority 


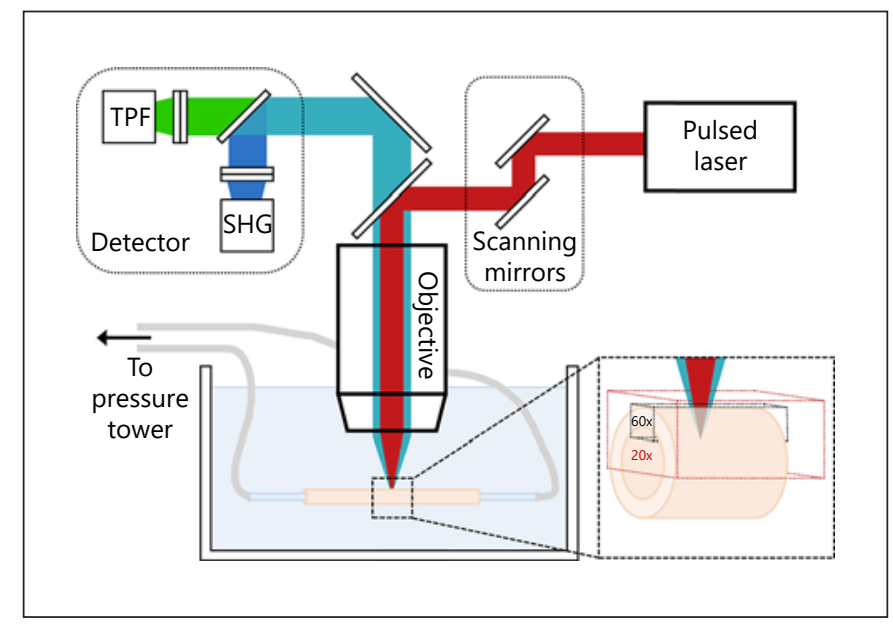

Fig. 1. Schematic of the experimental apparatus, with details of the myograph base and arms omitted for simplicity. The call out shows the approximate imaging volumes probed during the high resolution $(\times 60)$ and low resolution $(\times 20)$ protocols. TPF, two-photon fluorescence; SHG, second harmonic generation.

of the passive mechanical response occurs within the chosen pressure range [19]; and (iii) at higher pressures, the natural myogenic response of vessels becomes significant. Assessment of the passive mechanical response would necessitate elimination or suppression of vasoreactivity, losing the effect of basal tone (see e.g., [21]). One vessel was chosen at random from each group for imaging of cell nuclei stained with DAPI (Sigma), which was mixed into the myograph bath at a final concentration of $500 \mathrm{nM}$. For these vessels, the nuclear imaging replaced the final measurement at $50 \mathrm{~mm} \mathrm{Hg}$ transmural pressure. Additional low-resolution image stacks were acquired for all vessels at 3 and $30 \mathrm{~mm} \mathrm{Hg}$ to assess gross vessel morphology for fitting a mechanical model.

The multiphoton microscopy system [17] consisted of an upright modified confocal laser-scanning microscope (FluoView IX71 and F300; Olympus) and Ti:sapphire laser (Mira 900-D; Coherent) tuned to $816 \mathrm{~nm}$ and pumped by a $532 \mathrm{~nm}$ solid state laser (Verdi V10; Coherent) with a repetition rate of $76 \mathrm{MHz}$ and pulse width of $100 \mathrm{fs}$. Two-photon fluorescence (TPF) and second harmonic generation images were acquired simultaneously in the backwards direction, with signal corresponding to elastic fibres and collagen, respectively. Olympus UPlanSApo $20 \times 0.4 \mathrm{NA}$ and $60 \times 1$ NA water immersion objectives were used to acquire gross morphological and high-resolution images of immersed vessels at in-plane resolutions of 1.5 and $500 \mathrm{~nm}$, respectively. For high-resolution imaging, capture of each $512 \times 512$ pixel image took $22 \mathrm{~s}$, and approximately 120 images were acquired per pressure increment, at $1-\mu \mathrm{m}$ steps in the $z$-direction. The resulting $3 \mathrm{D}$ image stacks were $250 \times 250 \times 120 \mu \mathrm{m}$ in $x, y$, and $z$, respectively, and took $44 \mathrm{~min}$ to acquire. At the lower resolution, each $512 \times 512$ pixel image took $7 \mathrm{~s}$ to acquire, and approximately 100 images were acquired per pressure increment, at 2 - $\mu \mathrm{m}$ steps in the $z$-direction. The resulting $3 \mathrm{D}$ image stacks were $750 \times 750 \times 200 \mu \mathrm{m}$ in $x, y$, and $z$, respectively, and took $11 \mathrm{~min}$ to acquire.

Image stacks were converted into 3D images using the Volume Viewer plugin for Fiji [22]. The dimensions of each layer of the ves-
Table 1. Statistical summary of volunteer metrics and dimensions of the subcutaneous resistance arteries at $30 \mathrm{~mm} \mathrm{Hg}$ transmural pressure

\begin{tabular}{|c|c|c|}
\hline & Healthy & Diabetic \\
\hline Gender M/F & $8 / 5$ & $5 / 3$ \\
\hline $\mathrm{BMI}^{\dagger}$ & $24.5 \pm 2.5^{*}$ & $28.4 \pm 4.1^{*}$ \\
\hline Age, years ${ }^{\dagger}$ & $56.2 \pm 9.5$ & $63.1 \pm 13.7$ \\
\hline Systolic BP, mm Hg${ }^{\dagger}$ & $122.5 \pm 10.3$ & $128.8 \pm 12.0$ \\
\hline Diastolic BP, $\mathrm{mm} \mathrm{Hg}^{\dagger}$ & $74.5 \pm 7.6$ & $79.5 \pm 8.6$ \\
\hline Subcutaneous fat area, $\mathrm{mm}^{2 \dagger}, \ddagger$ & $19,100 \pm 6,900$ & $24,400 \pm 11,500$ \\
\hline Visceral fat, $\mathrm{mm}^{2 \dagger \ddagger}$ & $9,100 \pm 5,200^{* *}$ & $21,800 \pm 7,200^{* *}$ \\
\hline Years since T2DM diagnosis ${ }^{\dagger}$ & $\mathrm{N} / \mathrm{A}$ & $7.9 \pm 2.2$ \\
\hline $\mathrm{HbAlC}, \mathrm{mmol} / \mathrm{mol}$ & $36 \pm 4$ & $58 \pm 9$ \\
\hline Inner radius, $\mu \mathrm{m}$ & $111 \pm 16$ & $138 \pm 20$ \\
\hline Wall-lumen ratio (30 $\mathrm{mm} \mathrm{Hg}$ ) & $0.41 \pm 0.07^{* *}$ & $0.20 \pm 0.06^{* *}$ \\
\hline Adventitia-media ratio ${ }^{\S}$ & $0.82 \pm 0.11$ & $0.59 \pm 0.02^{\dagger}$ \\
\hline
\end{tabular}

Values for the wall-lumen ratio differ from those of other diabetic studies, as the vessels were measured at a comparatively low transmural pressure and did not undergo preconditioning prior to measurement. This arrangement allows the passive mechanical behaviour of vessels to be probed. BMI, body mass index; BP, blood pressure; T2DM, type 2 diabetes mellitus. * Statistically significant difference between healthy and diabetic groups $\left({ }^{*} p<0.05,{ }^{* *} p<0.01\right) .{ }^{\dagger}$ Mean $\pm \mathrm{SD} .{ }^{*}$ Fat area measurements used as indicator of fat compartment size. ${ }^{\S}$ Adventitia-media ratio only measured in subset of arteries from T2DM patients that exhibited distinct layers $(n=5)$.

sel walls were obtained by fitting circles to the layer boundaries at 5 longitudinal positions. Where the vessel morphology was not circular, the algorithm fitted a circle with an area matching to that of the region of interest. Two layers were defined for each vessel where possible: the intima-media, extending from the luminal surface to the outer edge of the cellular region (which appears dark under TPF and second harmonic generation); and the adventitia, which comprises the collagenous outer layer. Orientation distributions for the 3 fibrous protein networks (internal elastic lamina, adventitial elastic fibres, and collagen) were obtained with the OrientationJ Distribution plugin for Fiji [23]. Interfibrillar spacing in the internal elastic lamina (IEL) of each vessel was estimated by taking circumferential profiles of the TPF signal, and calculating the distance between full-width half-maximum positions for each fibre.

The relative movement of each boundary after changes in intramural pressure was used to inform a mechanical model that calculates the stiffness and stress in each layer. This model has been described previously [17] and is based upon linear thick-walled cylinder theory [24]. The model assumes negligible axial strain and torsion, which was deemed to be a reasonable assumption because the vessel ends were attached to the myograph glass capillaries. A second model calculating wall stress on the assumption that the vessel can be approximated as a homogeneous thick-walled tube, which is a generalized version of the simple "wall stress" model widely used to analyse myography data [25] was also utilized for comparison.

The amount of visceral and subcutaneous fat in the vicinity of the biopsy was measured prior to the procedure using MRI. Fat quantification was based on area measurements using established protocols [26-28]. 


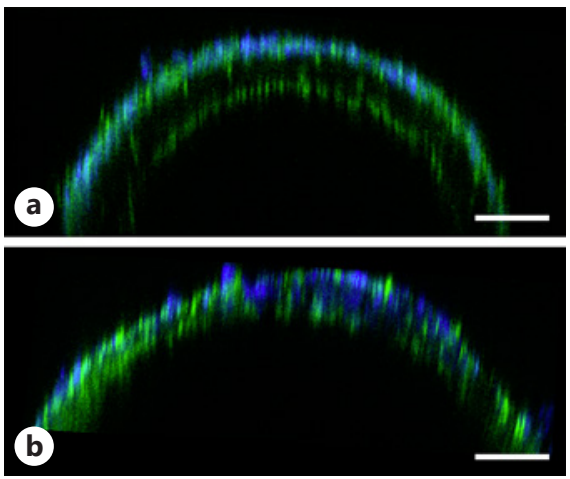

Fig. 2. Comparison of healthy and diabetic morphologies in section at $30 \mathrm{~mm} \mathrm{Hg}$ transmural pressure. False colours represent elastic fibres and intracellular proteins (green), and fibrous collagen (blue). a The healthy artery exhibits a distinct IEL, media and adventitia, and the wall thickness is relatively uniform. b The diabetic artery exhibits an IEL, but the adventitia and media overlap and are of uneven thickness. Bars $50 \mu \mathrm{m}$. T2DM, type 2 diabetes mellitus; IEL, internal elastic layer.

\section{Statistics}

Data are presented as mean \pm SEM unless otherwise stated. Statistical significance was calculated using the Mann-Whitney U test unless otherwise stated, with the null hypothesis rejected at the 5\% significance interval. Ranked correlations were calculated using Spearman's rho, with the null hypothesis rejected at the 5\% significance level. Data were analysed and presented using MATLAB (MathWorks).

\section{Results}

Resistance arteries were obtained from 13 healthy control and 8 T2DM subjects. The same number of T2DM subjects as controls were recruited, but the T2DM biopsies contained fewer blood vessels and were more friable than the controls, and therefore less likely to yield a suitable vessel, due to an absence of vasculature or damage. The T2DM group had a significantly higher BMI than the control group $(p<0.05)$. A summary of patient data and vessel dimensions is given in Table 1.

\section{Microscopic Observations}

There was considerable variation in the structure of vessels from the T2DM group. One vessel showed only minor distortion of the IEL, but the majority showed variations in wall thickness with breakdown of the layered structure and disorder of the fibrous protein networks, particularly in the adventitia as detailed below.

The control group vessels exhibited a clear 3-layered structure, comprising (from inside to outside) IEL, media
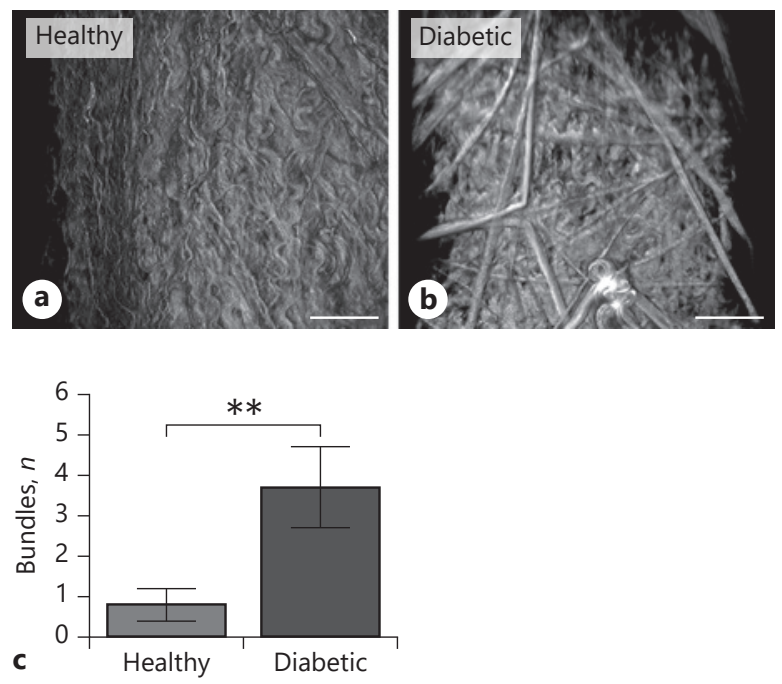

Fig. 3. Comparison of adventitial collagen morphology in health and diabetes mellitus, at $30 \mathrm{~mm} \mathrm{Hg}$ transmural pressure. a Healthy adventitia, comprising tortuous collagen fibre bundles of varying diameter and at varying stages of recruitment. b Diabetic adventitia, comprising straight, bracing collagen fibre bundles surrounding an interior layer of tightly wound tortuous collagen fibre bundles. c The number of bracing fibre bundles present within the imaged volume of healthy arteries and arteries from individuals with T2DM. There were significantly more in arteries from individuals with T2DM ( $p<0.01$, Mann-Whitney U test). Bars $50 \mu \mathrm{m}$. T2DM, type 2 diabetes mellitus

and adventitia when viewed in section (Fig. 2a), but the boundaries in some arteries from T2DM patients (3/8) were not clear (Fig. 2b), largely due to the appearance of matrix material in the media. This may represent a more advanced stage of remodelling. One artery exhibited matrix material in the media but still retained the layered structure. The other arteries from T2DM patients exhibited the same layered structure as controls, although overall the adventitia occupied a proportionally smaller proportion of the total wall volume in arteries from T2DM patients than controls. The presence of matrix material in the media did not correlate with any measures of abdominal fat content.

As vascular remodelling is commonly described in terms of increased collagen deposition leading to mechanical recruitment at lower transmural pressure, initial analysis examined the fibrous collagen of the adventitia. In normal vessels, the adventitia comprises helical fibre bundles of varying straightness that recruit under increases in pressure (Fig. 3a), but all except one of the arteries from T2DM patients showed significant quantities 


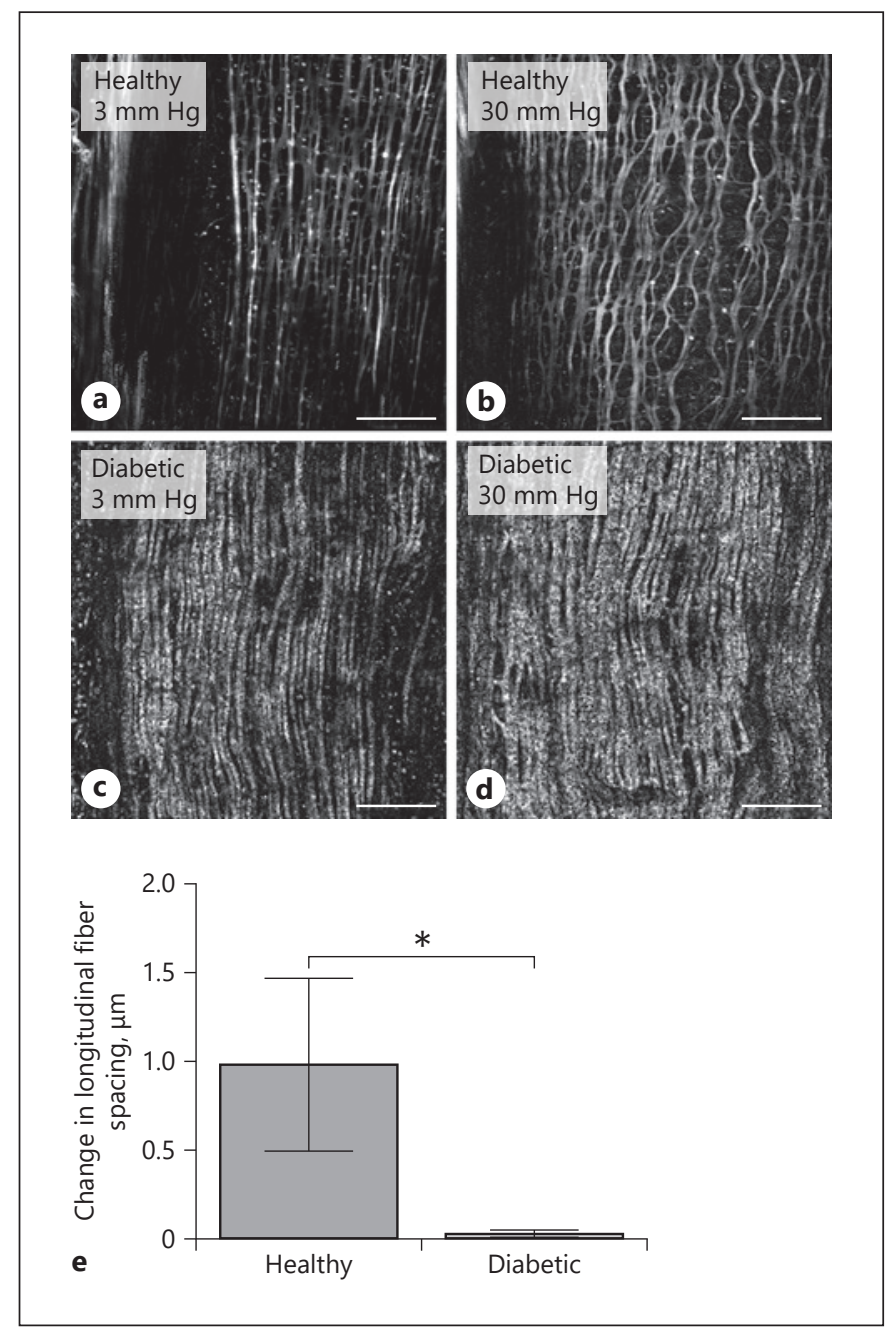

Fig. 4. Comparison of IEL morphology in health and diabetes mellitus. a The healthy IEL at 3-mm Hg transmural pressure comprises longitudinal elastic fibres, braced periodically by thinner fibres. b At $30-\mathrm{mm} \mathrm{Hg}$ transmural pressure the IEL distends considerably and deforms into a honeycomb structure. $\mathbf{c}$ The diabetic IEL at 3-mm $\mathrm{Hg}$ transmural pressure also comprises longitudinal fibres, but they appear kinked and uneven. d At 30-mm Hg transmural pressure, the IEL has distended, but not to the same degree as the healthy artery, and looks morphologically similar to the lowpressure case, except for in discrete positions where some fibres have moved apart. e Change in longitudinal elastic fibre spacing. The change in spacing in healthy arteries is significantly greater than in arteries from individuals with T2DM $\left({ }^{*} p<0.05\right)$, Mann Whitney U test. Bars $50 \mu \mathrm{m}$. IEL, internal elastic layer; T2DM, type 2 diabetes mellitus.

of thick, straight, bracing fibre bundles forming what might constitute a constraining sheath around the outer edge (Fig. 3b). Underneath these fibre bundles, the collagen network was morphologically similar to normal. The bracing fibre bundles were sparse and did not form continuous layers. The arteries from T2DM patients were more variable in diameter along their length than those from healthy volunteers (see online suppl. material for full details on diameter variability; for all online suppl. material, see www.karger.com/doi/10.1159/000517856), and more constricted regions often contained larger numbers of the bracing fibre bundles. Three of 8 arteries from T2DM patients exhibited additional bundles of normal collagen fibres outside the bracing fibres, which were unevenly distributed around the vessel circumference. This loose, peripheral collagen did not change in organization at any pressure increment and therefore did not appear to have any mechanical function, despite being clearly distinct from the neighbouring matrix. The network of adventitial elastic fibres was more disordered in arteries from T2DM patients than controls, but the fibres themselves were morphologically similar.

Bracing fibre bundles were found in 3 arteries from the control group, with $0.8 \pm 0.4$ bundles per imaged region (area $0.061 \mathrm{~mm}^{2}$ ) of each vessel. All but 1 artery from the diabetic cohort showed bracing fibre bundles, with $3.7 \pm$ 1.0 bundles within the imaged regions of each vessel. This difference was significant at the $p<0.01$ level (Fig. 3c). A Spearman's ranked correlation test showed that the number of fibre bundles were close to correlating with systolic blood pressure $(p=0.06)$ in the healthy cohort, but there was no correlation in the diabetic cohort, or when the 2 cohorts were merged (see online suppl. material for a full comparison of BP to number of bundles). The presence of bracing fibre bundles did not correlate with any measures of fat content for either cohort.

The IEL in healthy vessels comprises longitudinal elastic fibres that are interconnected by periodic, thinner branching fibres, which move apart to form a honeycomb network under pressure (Fig. 4a, b). The control IEL distended by $45 \pm 9 \%$ as transmural pressure was ramped from 3 to $30 \mathrm{~mm} \mathrm{Hg}$. In arteries from T2DM patients, the IEL appeared to twist about the vessel axis, and the longitudinal fibres undulated and clumped together rather than forming a uniform network as in the control tissues. Increasing luminal pressure caused less reorganization of the network than in controls, with the majority of longitudinal fibres remaining contorted and closely associated, despite the diabetic lumen distending by $33 \pm 5 \%$ (Fig. 4c, d). The average change in spacing between discrete longitudinal elastic fibres (Fig. 4e) in control vessels was 0.97 $\pm 0.49 \mu \mathrm{m}$, while in arteries from T2DM patients it was $0.03 \pm 0.02 \mu \mathrm{m}(p<0.01)$. This behaviour indicates that the IEL is compressed in T2DM, which may reflect the lack of distensibility of the outer layers. 
We hypothesized that the infiltration of fibrous protein into the media would have a significant effect upon the morphology and vasoactive ability of VSMCs in the media, so we applied DAPI nuclear stain to the bath of 1 healthy and 1 T2DM vessel in order to visualize the cell nuclei. Figure 5 shows the fluorescence from 3 cell types (red - endothelial cells; blue - VSMCs; yellow - fibroblasts, cell phenotype predicted by morphology and position in the vessel wall) overlaid upon TPF sections through the vessel wall (section position shown in inset) in health (Fig. 5a) and disease (Fig. 5b). The distribution of cell nuclei orientations are shown in Figure $5 \mathrm{c}$. In healthy resistance arteries, the endothelial cell nuclei were arranged close to longitudinal (standard deviation $16^{\circ}$ ), but in disease the orientation was much more random (standard deviation $32^{\circ}$ ). Given the association between endothelial cell morphology and fluid mechanics, this may indicate a change in haemodynamics in the diseased vessels. However, there was a similar loss of organization in the VSMCs. VSMC nuclei in health were arranged mostly parallel to one another $\left(\mathrm{SD} 9^{\circ}\right)$, and in a single or double layer. In diseased arteries, the VSMCs varied in orientation considerably along the length of the vessel (SD $20^{\circ}$ ).

\section{Mechanical Analysis}

In the following analysis, mechanical calculations are based upon the differences in vessel layer boundary positions at transmural pressures 3 and $30 \mathrm{~mm} \mathrm{Hg}$. The control group includes data from the study by authors in reference [17]. The layer boundaries were defined as the lumen, the transition from cellular media to fibrous adventitia, and the outer edge of the vessel wall (not including clumps of loose, unrecruited collagen that were found on the outer edge of some arteries from T2DM patients).

Changes in wall volume associated with increased transmural pressure are shown in Figure 6a. The small

Fig. 5. Two-photon fluorescence optical sections through healthy vessel walls and vessel walls from individuals with T2DM (optical plane depicted in inset), with DAPI-stained cell nuclei overlaid in false colour. a In health, endothelial cell nuclei (red) are arranged longitudinally, while VSMC nuclei (blue) are arranged circumferentially. Fibroblast nuclei (yellow) exhibit no preferential shape. b In the diabetic artery, the endothelial cell nuclei (red) appear more irregular in shape and orientation, while the VSMC nuclei (blue) retain a characteristic slender morphology but are arranged more randomly. c Distributions of endothelial cell and VSMC nuclei orientations, measured as an angle relative to the longitudinal vessel axis. Bars $50 \mu \mathrm{m}$. T2DM, type 2 diabetes mellitus; EC, endothelial cell; VSMC, vascular smooth muscle cell.

Microstructural Changes in Vascular Remodelling
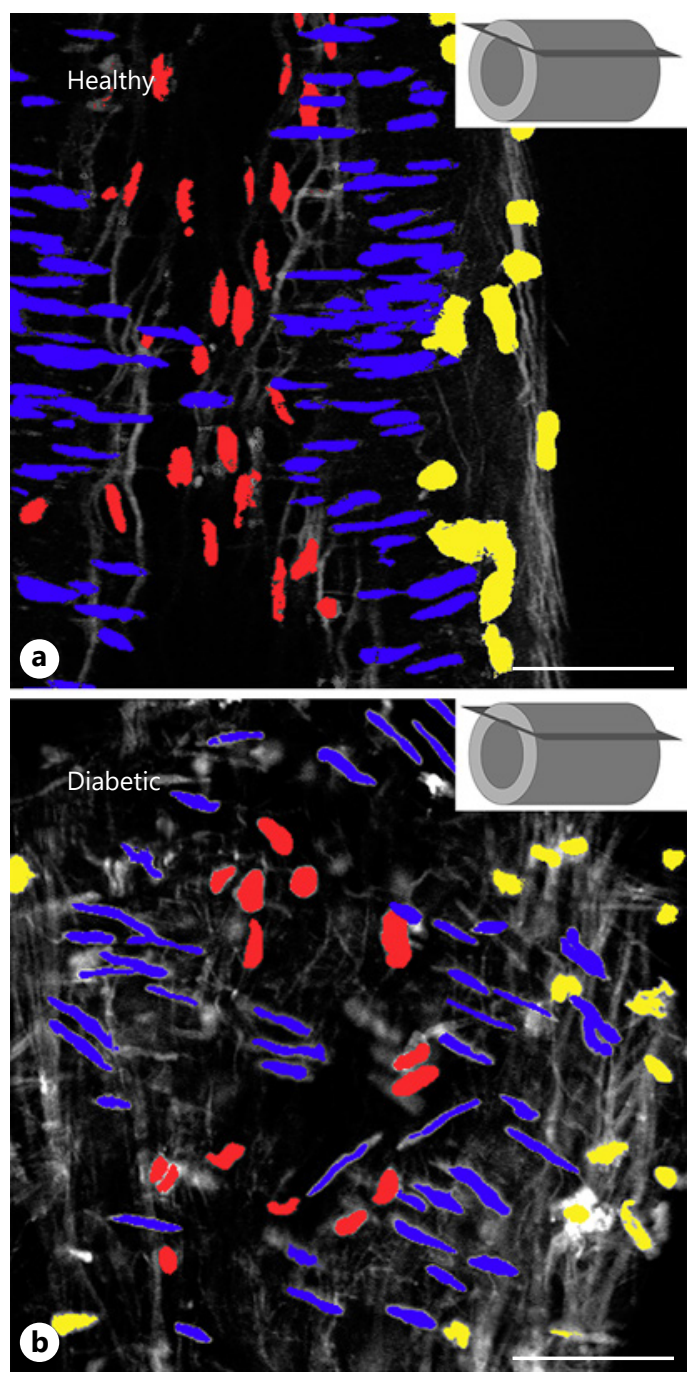

EC orientation

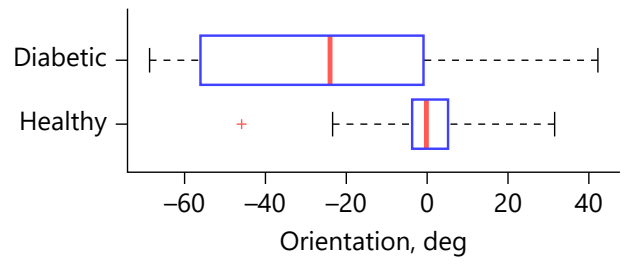

VSMC orientation

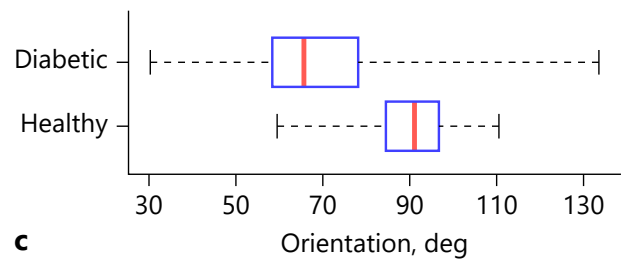

J Vasc Res 2022;59:50-60

DOI: $10.1159 / 000517856$ 


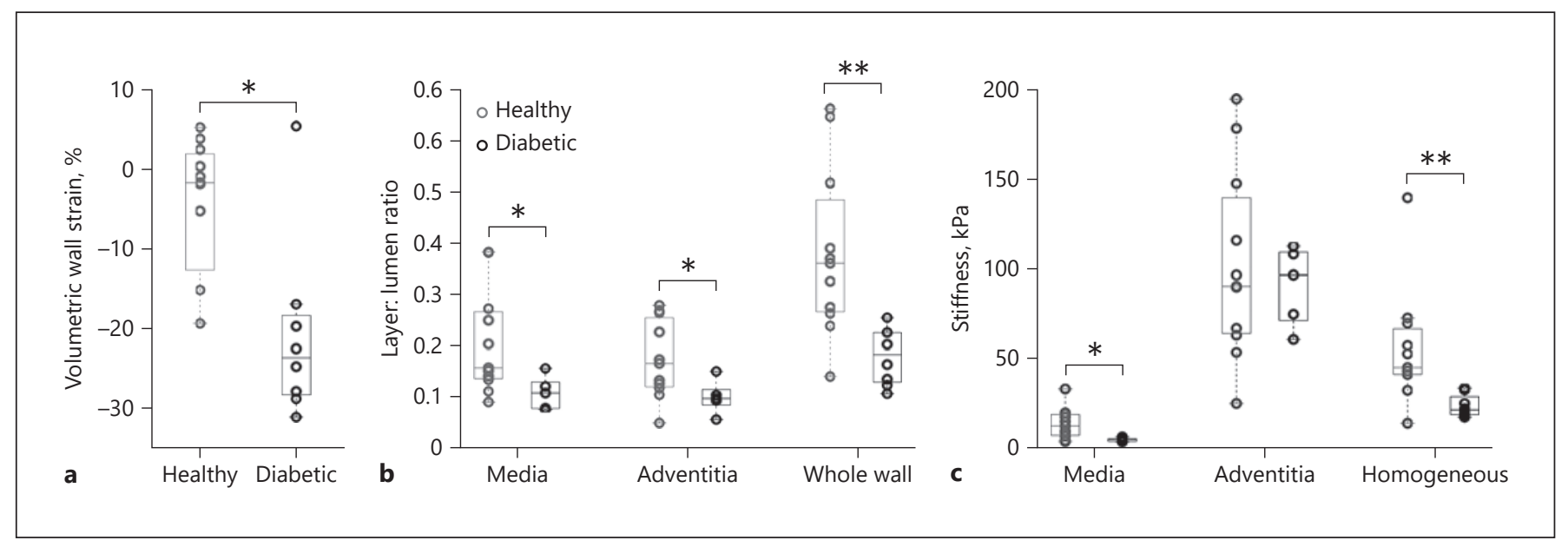

Fig. 6. Morphological and mechanical results. a Volumetric wall strain (analogous to change in cross-sectional area) in healthy and arteries from individuals with T2DM, following pressurization from 3 to $30 \mathrm{~mm} \mathrm{Hg}$. b Ratio of layer thickness to lumen radius at $30-\mathrm{mm} \mathrm{Hg}$ transmural pressure. c Elastic modulus of each layer, and of the entire wall assuming homogeneity, determined by elastic modelling fitted to experimentally determined deformations $\left({ }^{*} p<0.05,{ }^{* *} p<0.01\right)$. T2DM, type 2 diabetes mellitus.

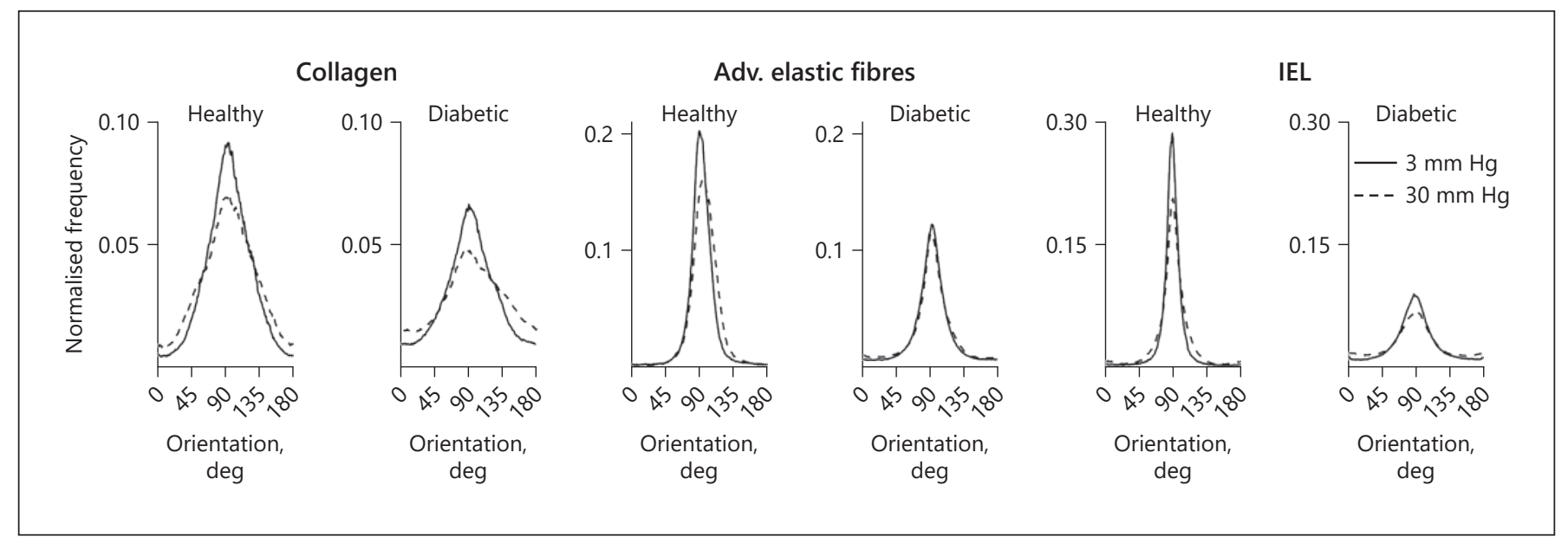

Fig. 7. Distribution of fibrous protein orientations in health and arteries from individuals with T2DM, at varying transmural pressure. T2DM, type 2 diabetes mellitus; Adv, adventitial.

average volume change in control vessels was confined to the adventitia, while in arteries from T2DM patients the volumes of both media and adventitia reduced significantly. The relative thickness of each layer at $30 \mathrm{~mm} \mathrm{Hg}$ transmural pressure is shown in Figure 6b, where both the media and adventitia are significantly thinner in T2DM than in health $(p<0.05)$. The large medial volume reduction in diabetes is due to a lower medial stiffness (Fig. 6c) than that of healthy arteries ( $3.8 \pm 0.6$ vs. $16.5 \pm$ $5.2 \mathrm{kPa}, p<0.05)$. The adventitia stiffness was lower in arteries from T2DM patients, but not significantly so (101 \pm 14 vs. $114 \pm 46 \mathrm{kPa}$ ). It should be noted that the model does not discriminate between the pathological and normal collagen, and so averages the contributions of both recruited and unrecruited fibres. The alternative homogeneous model found the arteries from T2DM patients to be less stiff ( $24 \pm 3$ vs. $71 \pm 19 \mathrm{kPa}, p<0.05)$.

Subcutaneous fat area was found to correlate with wall strain and adventitial layer strain in both cohorts separately $(p<0.05)$, but not when combined. It was also found to correlate inversely with adventitial stiffness in both cohorts separately $(p<0.05)$, but not when com- 
bined. No correlations were found with visceral fat or the ratio of subcutaneous to visceral fat.

Analysis of fibre organization confirmed the qualitative observations made above that the fibrous proteins in vessels from individuals with T2DM are less ordered than those in control vessels (Fig. 7). As luminal pressure increased, the shift in adventitial collagen and IEL elastin organization from a longitudinal to a more isotropic arrangement was similar in control and arteries from T2DM patients. The adventitial elastin network became more isotropic and developed a slight helical bias as pressure increased in control vessels, but in T2DM vessels, there was barely any change with pressure. The collagen network exhibited a shoulder at $135^{\circ}$ at the higher pressure, suggesting a potentially helical trend in the fibre recruitment.

\section{Discussion}

The microstructure of small human resistance arteries in control subjects and individuals with type 2 diabetes mellitus, and its passive response to changes in transmural pressure, has been investigated using multiphoton microscopy. The extent of vascular remodelling varied between subjects in the diabetic cohort, as would be expected in a chronic disease, and the arteries from individuals with T2DM appeared to exhibit 2 stages of remodelling. The sample size in this study was limited by the difficulties of subject recruitment and vessel isolation from biopsies; however, a number of structural features were consistently observed and the structural bases of previously reported changes in mechanical characteristics were revealed.

The most common feature in diabetic remodelled arteries was the presence of bracing collagen fibre bundles in the outer adventitia. These bundles were also found in 2 of the 11 control arteries, although significantly fewer in number, suggesting they may be a feature of vascular remodelling in general. The presence of bracing fibre bundles is indicative of inward remodelling, as each of the layers underneath appeared compressed and distorted, and the ability to distend passively was significantly hindered in comparison to control arteries. Increasing transmural pressure had the effect of reducing wall volume significantly in T2DM, while in contrast healthy arteries did not change in wall volume. The bracing fibre bundles appear to form a stiff outer sheath that limits distensibility, and that the underlying layers press and deform against in order to accommodate pressure-induced changes in lumen diameter. Underneath the bracing fibre bundles, the adventitial collagen appeared morphologically normal; however, it did not recruit at any pressure increment, whereas in health, a significant portion of the adventitial collagen has been shown to recruit at $20 \mathrm{~mm} \mathrm{Hg}$ [18]. This would suggest that normal adventitial collagen in arteries from T2DM patients does not contribute to arterial mechanics in the same way as in healthy arteries.

While healthy resistance arteries exhibit a distinct layered structure of fibrous adventitia, cellular media and a single lamella intima, some arteries from T2DM patients exhibited infiltration of collagen fibres into the media, leading to blurring of the layer boundaries. In 3 of the 8 arteries from T2DM patients, sufficient collagen had been deposited to lose the layered structure altogether. All vessels that exhibited medial fibrosis also presented bracing fibre bundles, but conversely there were several arteries exhibiting bracing fibre bundles without medial fibrosis. It would appear, therefore, that the formation of bracing fibre bundles precedes medial fibrosis. Increased collagen:elastin ratio is a commonly reported feature of microvascular remodelling in both hypertension $[29,30]$ and diabetes [31], and the deposition of medial collagen could be associated with fibroblast migration, as has been reported in large arteries [32]. Quantification of collagen and elastin absolute volume is not possible to perform accurately using multiphoton microscopy due to many of the fibrous structures being significantly below the diffraction limit, leading to large errors (the morphological and structural observations in this study, on the other hand, are accurate to an error of approximately $1 \mu \mathrm{m})$. Remodelling in large arteries is suggested to be driven by mechanosensing in adventitial fibroblasts [33] and smooth muscle cells, for the purpose of reducing circumferential and shear stresses [34]. It is possible therefore that the collagen deposition observed in this study is a result of a previously altered local mechanical environment associated with the formation of bracing fibre bundles.

It has been shown previously that small arteries from T2DM patients have reduced wall stiffness compared to those from healthy volunteers [25]. The homogeneous model used in this study mimics those used elsewhere in the literature, and it too concludes that the wall stiffness, taken as a whole, reduces in T2DM. However, this result belies the significant redistribution of stress and strain within the vascular wall in T2DM, which can only be elucidated by adopting a more sophisticated model, as used in this study. While the bracing fibre bundles appear to constrain the outer edge of T2DM (and some healthy) arteries, reducing their ability to distend, the significant mechanical difference is found in the media, which in T2DM is less than a quarter as stiff as in healthy controls. 
Fig. 8. Illustrations of vessel cross-sections, showing undeformed geometry (left) and deformed geometry (right). Arrows indicate predicted direction of local stress associated with transmural pressure. a, b In the healthy resistance artery the adventitia allows outward distension whilst gradually stiffening due to collagen recruitment. The significantly more compliant media distends with the adventitia, thins as the adventitia stiffens, and resists a small portion of the circumferential stress. c, d Vessels exhibiting the first stage of remodelling (constraining fibre bundles only) are less able to distend their outer edge, and so the underlying normal adventitia does not recruit. Circumferential stress is therefore limited to the pathological fibre bundles in the periphery, and the rest of the vessel wall is squeezed up against the stiff outer edge by the transmural pressure. e, f Vessels exhibiting the second stage of remodelling (constraining fibre bundles and media fibrosis) are assumed to behave more like homogeneous pipes. The presence of collagen in the inner layers shifts the distribution of circumferential stress away from the periphery and into a more uniform state.

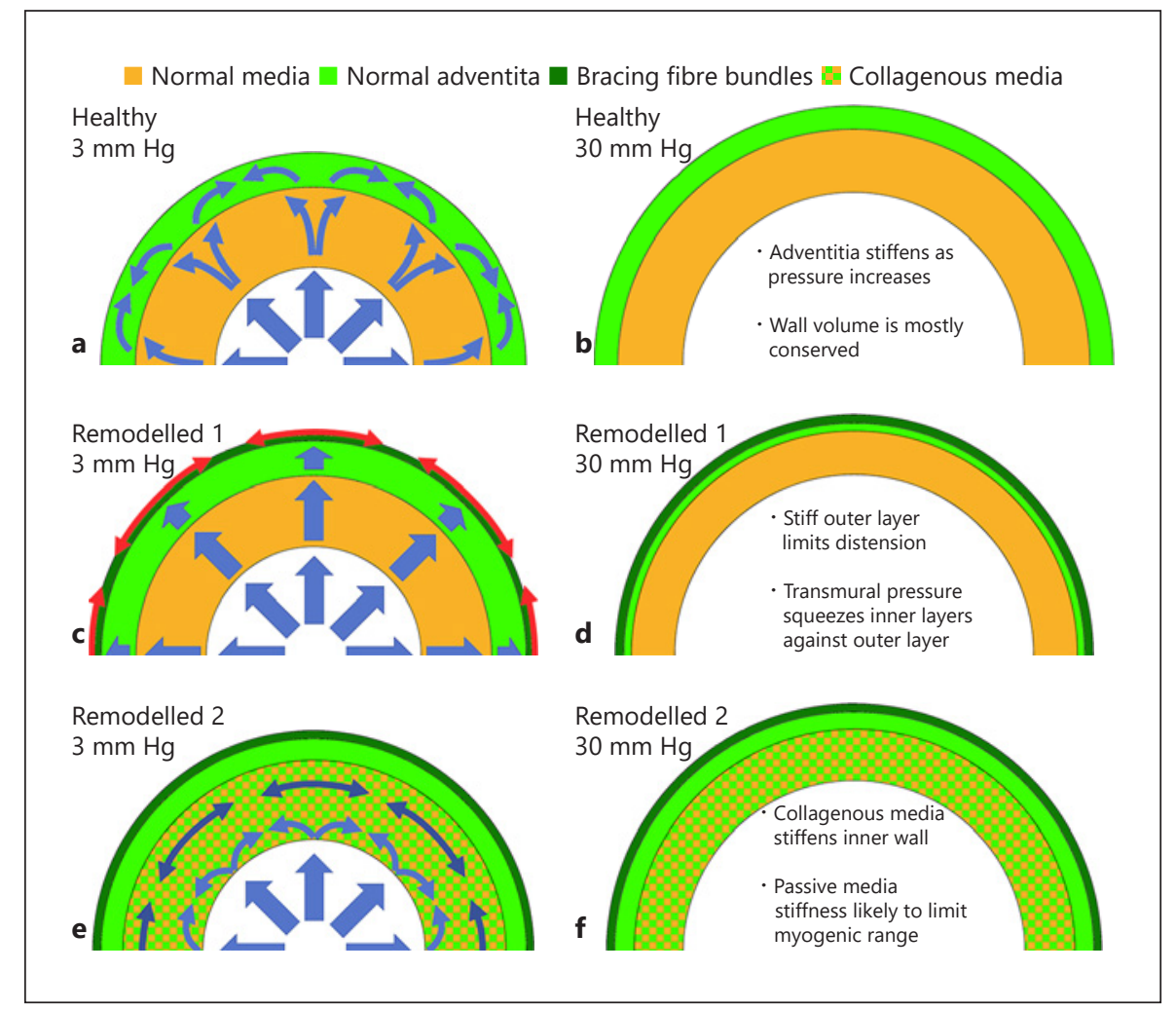

Our previous work has shown that in health, the adventitia bears between 50 and $90 \%$ of the circumferential stress, with the exact amount scaling with vessel radius and transmural pressure [17]. The rest is borne by the IEL and basal tone in the media. This study has shown that in arteries from individuals with T2DM, who exhibit bracing fibre bundles but with an intact layered structure, the adventitia bears between 90 and $95 \%$ of the circumferential stress (most of it likely taken up by the bracing fibre bundles). The IEL and media bear on average less than a third of the stress of the same regions in healthy vessels of comparable geometry, likely due to a decrease in basal tone [25]. In the more remodelled arteries from T2DM patients, which exhibit both bracing fibres and significant quantities of medial collagen, if the additional collagen is load bearing then it will have a homogenizing effect on the mechanical properties. A homogeneous, thick-walled pipe under luminal pressure experiences highest stress in the lumen and so as more load-bearing collagen is deposited in the media; the distribution of stress becomes more uniform. It is possible, then, that fibrosis is a passive compensating mechanism for a reduction in basal tone; however, the presence of collagen in the media is likely to exacerbate this reduction, by restricting the range over which VSMCs can deform. The distribution of stress in vessels at the different stages of remodelling observed in this study is described diagrammatically in Figure 8.

It was noted qualitatively during the sample preparation that diabetic adipose tissue samples were much more friable than the healthy samples. Given that the adventitia serves in part as an anchor to surrounding tissue, it can be reasonably expected that the reduction in support from the surrounding tissue will necessitate a structural response. A follow-up study that compares the mechanical properties of arteries with their surrounding tissue could yield intriguing results. The most significant structural change in T2DM, the formation of bracing fibre bundles, did not correlate with any fat area metrics. However, the correlations between adventitial stiffness and strain with subcutaneous fat area suggest a mechanical coupling of some form.

Disorder of extracellular matrix fibres and cell nuclei were most severe in the heavily remodelled arteries. The extent of disorder was not completely random; however, with both measurements of collagen fibre and cell nucleus orientation, exhibiting a helical preference. In pressurized large arteries there is a tendency for adventitial collagen to reorientate towards $\pm 45^{\circ}$ to the longitudinal vessel axis 
[35], reflecting the local force distribution [36], and while the diabetic adventitial collagen in this study exhibits similar behaviour, it is biased towards a particular helical handedness. Similarly, the VSMC nuclei also exhibit a preferential helical orientation following remodelling. This breakdown of symmetry in both passive and active components of the vessel wall will cause torsion. Increased tortuosity of microvessels is commonly reported with vascular remodelling, such as in diabetic retinopathy [9] and pulmonary hypertension [37], and the images acquired in this study indicate a potential microstructural basis.

We noted above the difference in intramural strain fields in healthy arteries and arteries from T2DM patients and this has important implications for VSMC function. The constraint of the adventitial collagen in the arteries from T2DM patients forces the VSMCs to accommodate a much greater proportion of lumen distension due to changes in transmural pressure - they essentially get compressed against the adventitia. Furthermore, given that most adventitial collagen is not recruited in diseased vessels during inflation, the VSMCs may not have a stable foundation to pull against to generate changes in vascular tone. This may explain at least in part the reduction in response to acetylcholine and bradykinin reported in diabetic resistance arteries [31], and their impaired myogenic response to transmural pressure [25]. The distorted alignment of VSMCs in diabetes is likely to further restrict their ability to regulate vascular tone. That the EC arrangement is also distorted in diabetes would suggest that the aligning effect of blood flow shear is dominated by the compressive disordering associated with inward remodelling. Deviation from parallel alignment is likely to have a negative effect on shear sensing. The disorder measured in the cellular networks in diabetes is potentially of great importance and warrants further study.

There are several ways this study could be taken further in the future. Firstly, to isolate the mechanical effect of changes in the extracellular matrix caused by T2DM, such as the increase in abundance of bracing collagen fibre bundles, vasodilating agents could be used to remove contributions from vascular tone. Conversely, while we note above that medial fibrosis likely impinges on VSMC ability to regulate tone, the use of vasoconstrictors, like noradrenaline, in conjunction with the imaging techniques presented here could provide a direct and accurate measure of the myogenic capabilities of vessels with and without medial fibrosis. The lack of statistical correlation between health metrics, such as duration and severity of T2DM and the experimental metrics obtained in this study may simply be due to insufficient statistical power.
Specifically, the Spearman's rank correlation with $p=$ 0.06 between systolic blood pressure and number of bracing fibre bundles in healthy individuals would be worthy of further investigation in studies focussed more on hypertension. Raw data for these measurements are included as online suppl. material for this purpose. Lastly, while the ambition had been for this study to age match the healthy and T2DM groups, difficulty with recruitment and sample preparation meant this was not feasible.

In conclusion, we have found 2 distinct microstructural changes that occur in vascular remodelling associated with diabetes mellitus. The first is the formation of thick, straight collagen fibre bundles, which brace the outer adventitia, providing increased resistance to distension. The second is fibrosis of the media, which is likely to have the effect of rebalancing the stress distribution across the vessel wall. The stiffness of arteries from individuals with T2DM was lower than controls, suggesting the increased quantity of collagen does not fully compensate for reduced basal tone. The techniques reported in this study could be used in conjunction with vasoactive species to elucidate the relationship between vascular remodelling and myogenic dysfunction in diabetes, as well as the therapeutic effects of antihypertensive treatment.

\section{Statement of Ethics}

Fully informed written consent was obtained in accordance with the Declaration of Helsinki. Ethics approval was granted by the NRES Committee South West, Exeter (11/SW/0199).

\section{Conflict of Interest Statement}

The authors have no conflicts of interest to declare.

\section{Funding Sources}

The presented research was funded by British Heart Foundation Grant No. PG/11/17/28788 and Medical Research Council Programme Grant MR/S037829/1 and was supported by the National Institute for Health Research (NIHR) Exeter Clinical Research Facility.

\section{Author Contributions}

J.S.B. designed the myograph, carried out the non-linear microscopy, analysed the images, wrote the analytical model, and drafted the manuscript. A.O.A. carried out vessel dissection and mounting and assisted with manuscript preparation. A.P. assisted 
in volunteer recruitment and screening and obtained biopsies. L.H. carried out volunteer recruitment and screening. C.E.T., A.C.S., and J.L.W. provided supervision and clinical interpretation of results, assisted with manuscript preparation, and raised funds for the study. C.P.W. provided supervision and physical interpretation of results, assisted with manuscript preparation, and raised funds for the study.

\section{References}

1 Cavan D, da Rocha Fernandes J, Makaroff L, Ogurtsova K, Webber S, editors. IDF diabetes atlas. 7th ed.; 2015.

2 Rizzoni D, Rosei EA. Small artery remodeling in diabetes mellitus. Nutr Metab Cardiovasc Dis. 2009;19(8):587-92.

3 Mulvany MJ. Small artery remodelling in hypertension. Basic Clin Pharmacol Toxicol. 2012;110(1):49-55

4 Grassi G, Seravalle G, Scopelliti F, Dell'Oro R, Fattori L, Quarti-Trevano F, et al. Structural and functional alterations of subcutaneous small resistance arteries in severe human obesity. Obesity. 2010;18(1):92-8.

5 Virdis A, Savoia C, Grassi G, Lembo G, Vecchione $\mathrm{C}$, Seravalle $\mathrm{G}$, et al. Evaluation of microvascular structure in humans: a "state-ofthe-art" document of the Working Group on Macrovascular and Microvascular Alterations of the Italian Society of Arterial Hypertension. J Hypertens. 2014;32:2120-9.

6 Cernes R, Zimlichman R, Shargorodsky M. Arterial elasticity in cardiovascular disease: focus on hypertension, metabolic syndrome and diabetes. Adv Cardiol. 2008;45:65-81.

7 Bruno RM, Duranti E, Ippolito C, Segnani C, Bernardini N, Di Candio G, et al. Different impact of essential hypertension on structural and functional age-related vascular changes. Hypertension. 2017;69(1):71-8.

8 Rizzoni D, De Ciuceis C, Porteri E, Semeraro $\mathrm{F}$, Rosei EA. Structural alterations in small resistance arteries in obesity. Basic Clin Pharmacol Toxicol. 2012;110(1):56-62.

9 Sasongko MB, Wong TY, Nguyen TT, Cheung CY, Shaw JE, Wang JJ. Retinal vascular tortuosity in persons with diabetes and diabetic retinopathy. Diabetologia. 2011;54(9):2409-16.

10 Niestrawska JA, Ch Haspinger DD, Holzapfel GA. The influence of fiber dispersion on the mechanical response of aortic tissues in health and disease: a computational study. Comput Methods Biomech Biomed Engin. 2018;21(2): 99-112.

11 Rafuse M, Xu X, Stenmark K, Neu CP, Yin X, Tan W. Layer-specific arterial micromechanics and microstructure: influences of age, anatomical location, and processing technique. J Biomech. 2019;88:113-21.

12 VanBavel E, Siersma P, Spaan JA. Elasticity of passive blood vessels: a new concept. Am J Physiol Heart Circ Physiol. 2003;285(5): H1986-2000.

13 Chow MJ, Turcotte R, Lin CP, Zhang Y. Arterial extracellular matrix: a mechanobiological study of the contributions and interactions of elastin and collagen. Biophys J. 2014;106(12): 2684-92.
14 Wang R, Brewster LP, Gleason RL. In-situ characterization of the uncrimping process of arterial collagen fibers using two-photon confocal microscopy and digital image correlation. J Biomech. 2013;46(15):2726-9.

15 Green EM, Mansfield JC, Bell JS, Winlove CP. The structure and micromechanics of elastic tissue. Interface Focus. 2014;4(2):20130058.

16 Roach MR, Burton AC. The reason for the shape of the distensibility curves of arteries. Can J Biochem Physiol. 1957;35(8):681-90.

17 Bell JS, Adio AO, Pitt A, Hayman L, Thorn $\mathrm{CE}$, Shore AC, et al. Microstructure and mechanics of human resistance arteries. Am J Physiol Heart Circ Physiol. 2016;311(6): H1560-8

18 Bloksgaard M, Leurgans TM, Nissen I, Jensen PS, Hansen ML, Brewer JR, et al. Elastin organization in pig and cardiovascular disease patients' pericardial resistance arteries. J Vasc Res. 2015;52(1):1-11.

19 Bloksgaard M, Leurgans TM, Spronck B, Heusinkveld MHG, Thorsted B, Rosenstand $\mathrm{K}$, et al. Imaging and modeling of acute pressure-induced changes of collagen and elastin microarchitectures in pig and human resistance arteries. Am J Physiol Heart Circ Physiol. 2017;313(1):H164-78.

20 Clifford PS, Ella SR, Stupica AJ, Nourian Z, Li M, Martinez-Lemus LA, et al. Spatial distribution and mechanical function of elastin in resistance arteries: a role in bearing longitudinal stress. Arterioscler Thromb Vasc Biol. 2011; 31(12):2889-96.

21 Khavandi K, Greenstein AS, Sonoyama K, Withers S, Price A, Malik RA, et al. Myogenic tone and small artery remodelling: insight into diabetic nephropathy. Nephrol Dial Transplant. 2009;24(2):361-9.

22 Schindelin J, Arganda-Carreras I, Frise E, Kaynig V, Longair M, Pietzsch T, et al. Fiji: an open-source platform for biological-image analysis. Nat Methods. 2012;9(7):676-82.

23 Rezakhaniha R, Agianniotis A, Schrauwen JT, Griffa A, Sage D, Bouten CV, et al. Experimental investigation of collagen waviness and orientation in the arterial adventitia using confocal laser scanning microscopy. Biomech Model Mechanobiol. 2012;11(3-4):461-73.

24 Vedeld K, Sollund HA, Hellesland J. Closed analytical expressions for stress distributions in two-layer cylinders and their application to offshore lined and clad pipes. J Offshore Mech Arct Eng. 2015;137:21702.

25 Schofield I, Malik R, Izzard A, Austin C, Heagerty A. Vascular structural and functional changes in type 2 diabetes mellitus: evidence for the roles of abnormal myogenic respon- siveness and dyslipidemia. Circulation. 2002; 106(24):3037-43.

26 Demerath EW, Shen W, Lee M, Choh AC, Czerwinski SA, Siervogel RM, et al. Approximation of total visceral adipose tissue with a single magnetic resonance image. Am J Clin Nutr. 2007;85(2):362-8.

27 Shen W, Punyanitya M, Chen J, Gallagher D, Albu J, Pi-Sunyer X, et al. Visceral adipose tissue: relationships between single slice areas at different locations and obesity-related health risks. Int J Obes. 2006;31(5):763.

28 Shen W, Punyanitya M, Wang Z, Gallagher D, St-Onge MP, Albu J, et al. Visceral adipose tissue: relations between single-slice areas and total volume. Am J Clin Nutr. 2004;80:271-8.

29 Intengan HD, Schiffrin EL. Structure and mechanical properties of resistance arteries in hypertension : role of adhesion molecules and extracellular matrix determinants. Hypertension. 2000;36:312-8.

30 Intengan HD, Thibault G, Li JS, Schiffrin EL. Resistance artery mechanics, structure, and extracellular components in spontaneously hypertensive rats : effects of angiotensin receptor antagonism and converting enzyme inhibition. Circulation. 1999;100(22):226775.

31 Rizzoni D, Porteri E, Guelfi D, Muiesan ML, Valentini U, Cimino A, et al. Structural alterations in subcutaneous small arteries of normotensive and hypertensive patients with non-insulin-dependent diabetes mellitus. Circulation. 2001;103(9):1238-44.

32 Zalewski A, Shi Y, Johnson AG. Diverse origin of intimal cells. Circ Res. 2002;91(8):6525.

33 Stenmark KR, Davie N, Frid M, Gerasimovskaya E, Das M. Role of the adventitia in pulmonary vascular remodeling. Physiology. 2006;21(2):134-45.

34 Humphrey JD. Mechanisms of arterial remodeling in hypertension: coupled roles of wall shear and intramural stress. Hypertension. 2008;52(2): 195-200.

35 Timmins LH, Wu Q, Yeh AT, Moore JE, Greenwald SE. Structural inhomogeneity and fiber orientation in the inner arterial media. Am J Physiol Heart Circ Physiol. 2010;298(5): H1537-45.

36 Hariton I, de Botton G, Gasser TC, Holzapfel GA. Stress-driven collagen fiber remodeling in arterial walls. Biomech Model Mechanobiol. 2007;6(3):163-75

37 Dababneh L, Cikach F, Alkukhun L, Dweik RA, Tonelli AR. Sublingual microcirculation in pulmonary arterial hypertension. Ann Am Thorac Soc. 2014;11(4):504-12. 